\title{
Adaptive Algorithms for Bayesian Spectrum Sensing Based on Markov Model
}

\author{
Shengliang Peng ${ }^{12}$, Renyang Gao ${ }^{1}$, Weibin Zheng ${ }^{1}$, Kejun Lei $^{3}$ \\ ${ }^{1}$ Xiamen Mobile and Multimedia Communications Key Laboratory, Huaqiao University \\ Xiamen 361021, China \\ ${ }^{2}$ Department of Electrical and Computer Engineering, Stevens Institute of Technology \\ Hoboken, 07030, USA \\ 3 College of Information Science and Engineering, Jishou University \\ Jishou 416000, China \\ *Corresponding author: Shengliang Peng \\ [e-mail: peng.shengliang@gmail.com]
}

Received August 4, 2017; revised October 15, 2017; accepted March 20, 2018;

published July 31, 2018

\begin{abstract}
Spectrum sensing (SS) is one of the fundamental tasks for cognitive radio. In SS, decisions can be made via comparing the test statistics with a threshold. Conventional adaptive algorithms for SS usually adjust their thresholds according to the radio environment. This paper concentrates on the issue of adaptive SS whose threshold is adjusted based on the Markovian behavior of primary user (PU). Moreover, Bayesian cost is adopted as the performance metric to achieve a trade-off between false alarm and missed detection probabilities. Two novel adaptive algorithms, including Markov Bayesian energy detection (MBED) algorithm and IMBED (improved MBED) algorithm, are proposed. Both algorithms model the behavior of PU as a two-state Markov process, with which their thresholds are adaptively adjusted according to the detection results at previous slots. Compared with the existing Bayesian energy detection (BED) algorithm, MBED algorithm can achieve lower Bayesian cost, especially in high signal-to-noise ratio (SNR) regime. Furthermore, it has the advantage of low computational complexity. IMBED algorithm is proposed to alleviate the side effects of detection errors at previous slots. It can reduce Bayesian cost more significantly and in a wider SNR region. Simulation results are provided to illustrate the effectiveness and efficiencies of both algorithms.
\end{abstract}

Keywords: Spectrum sensing, Markov model, Bayesian cost, Adaptive threshold 


\section{Introduction}

In recent years, with the development of wireless communications technologies, most spectrum bands have been allocated for specific use, and the spectrum resource is on the verge of extinction. However, many researchers found that significant amount of spectrum remains underutilized, or even completely idle [1-2]. Coined by Mitola [3], cognitive radio (CR) was proposed as a promising solution to this paradox via recycling the underutilized spectrum bands.

In a CR system, primary user (PU) is the licensed user who has priority to use the spectrum band, and secondary user (SU) is the unlicensed user who monitors the usage of spectrum band and opportunistically accesses the unutilized band without causing interference to PU [4]. To achieve this goal, SU should continuously and reliably sense whether the spectrum band is occupied by PU. There are several classical spectrum sensing (SS) methods, such as matched filter detection [5], cyclostationary feature detection [6] and energy detection (ED) [7]. Among them, ED is the simplest and the most popular SS mechanism since it does not require prior information about PU signals [8-9]. In [10-12], some cooperative PU detection approaches are suggested to exploit the diversity of multiple SUs.

Performance of SS is usually evaluated in terms of the false alarm probability and the missed detection probability. The former denotes the probability with which the absence state of PU is detected as presence, and the latter is the probability with which the presence state of PU is regarded as absence [13]. In ED, PU's state is decided by comparing the received energy with a predetermined threshold [14]. Consequently, how to determine a threshold is of great importance. The threshold can be chosen under a constant detection rate (CDR) criterion to ensure sufficient protection to PU. But when the signal to interference plus noise ratio (SINR) is larger, the blind use of CDR criterion probably incurs over-protection of PU and a high false alarm probability. In [13], an interference-aware approach was proposed to select threshold according to the distance between secondary transmitter and primary receiver. In order to enhance the sensing accuracy and robustness, reference [15] designed an adaptive threshold architecture, whose sensing component employed two SS techniques and machine learning component dynamically adopted the threshold based on the lowest Bayesian risk. Considering the coexistence of PU and SU signals, reference [16] adaptively changed its threshold according to the transmission power of SU to guarantee the minimum decodable SINR for primary receiver. In [17] and [18], the threshold was formulated as a linear increasing function of the instantaneous SINR. By means of estimating the noise power and signal power, reference [19] dynamically adapted its threshold based on the noise fluctuation, and derived an optimal threshold in function of the weighted error probability. Via minimizing the total error decision probability at different spectrum utilizations of PU, reference [20] proposed an adaptive threshold algorithm to achieve an efficient trade-off between the detection and false alarm probabilities.

Besides the adaptive SS methods listed above, some research work utilized the characteristic of PU's behavior to aid SS. The M-slot temporal persistence that PU maintains the same state for at least M slots was considered to improve the sensing accuracy [21]. Similarly, Zhao discussed the Markovian behavior of PU and a partially observable decision framework to maximize the throughput of CR system [22]. In [23], the authors proposed some cognitive medium access control (MAC) protocols to optimize the performance of SUs based on a partially observable Markov decision process framework. In order to predict the behavior 
of PU more accurately, actual measurements in the $2.4 \mathrm{GHz}$ industrial, scientific and medical (ISM) band with a vector signal analyzer were performed, indicating a continuous-time semi-Markov model [24]. The sojourn time of this model was also characterized, and the channel was categorized by five different states [25]. In order to simplify the semi-Markov model, Zhao considered a myopic policy to maximize the immediate reward and ignore the impact of current state on the future reward. When the channels are independently and identically distributed (i.i.d.), the myopic sensing policy has a simple and robust structure, and the model can be regarded as a two-state process [26].

Although the existing adaptive SS methods are able to improve sensing performance, they adjust their thresholds according to the wireless environment [16-19], such as signal power or noise level, which not only requires environmental parameter estimation but also suffers from estimation errors. On the other hand, despite the existence of research efforts on the behavior of PU, the temporal persistence model in [21] is not widely followed by other researchers, and the popular Markov models are mainly utilized to enhance system throughputs [22-23, 26], while their benefits to other performance metrics have not been fully investigated.

This paper focuses on the Markovian behavior of PU, and utilizes this characteristic to achieve an efficient trade-off between false alarm and missed detection probabilities. Some adaptive threshold algorithms are consequently proposed. Unlike the existing methods that adaptively adjust thresholds according to the wireless environment, our algorithms make adjustment based on the behavior of PU. Our work is also different from previous research on the behavior of PU because we use a two-state Markov model to reduce the total cost of false alarm and missed detection instead of increasing system throughputs.

Two algorithms, namely Markov Bayesian energy detection (MBED) and improved MBED (IMBED), are consequently proposed. Both algorithms exploit the behavior of PU to reduce the total cost of detection errors. In MBED algorithm, the threshold is chosen from two possibilities according to previous detection results. In IMBED algorithm, the threshold is recalculated based on the posterior probabilities of previous detection.

The remainder of this paper is organized as follows: System model and conventional ED method are described in Section 2. In Section 3, optimal threshold for Bayesian energy detection (BED) algorithm is re-derived, the benefit of Markov model is analyzed, and the MBED algorithm is proposed. Section 4 presents the IMBED algorithm to alleviate the side effect of detection errors. Simulation results are provided in section 5 and conclusions are drawn in section 6 .

\section{System Model}

Consider that the behavior of PU can be described as a two-state discrete-time Markov Model with the transition probability $q$ and $p$ [26], as shown in Fig. 1. Detailedly, $q$ is the probability with which the state of $\mathrm{PU}$ changes from absence to presence, and $p$ is that from presence to absence. Then, the prior probabilities of PU's presence (i.e., $P_{1}$ ) and absence (i.e., $P_{0}$ ) are given by $[17,18]$

$$
\left\{\begin{array}{l}
P_{0}=\frac{p}{p+q} \\
P_{1}=\frac{q}{p+q}
\end{array} .\right.
$$




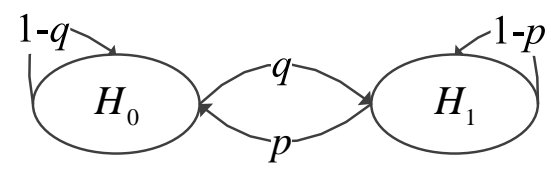

Fig. 1. Markov model of PU's behavior

Without loss of generality, a slotted CR system consisting of one PU and one SU is considered in this paper. SU performs ED at each slot to continuously sense the state of PU, which can be regarded as a binary hypothesis problem as follows,

$$
y_{i}(k)=\left\{\begin{array}{ll}
n_{i}(k) & H_{0} \\
s_{i}(k)+n_{i}(k) & H_{1}
\end{array},\right.
$$

where $y_{i}(k)$ is the signal received by SU at the $i$ th slot; $n_{i}(k)$ denotes the additive white Gaussian noise (AWGN) at the $i$ th slot with zero mean and variance of $\sigma_{n}^{2} ; s_{i}(k)$ is the PU signal at the $i$ th slot and assumed to be i.i.d. with zero mean and variance of $\sigma_{s}^{2} ; H_{0}$ and $H_{1}$ denote the hypotheses of PU's absence and presence, respectively. As a result, the received energy at the $i$ th slot can be calculated as

$$
v_{i}=\sum_{k=1}^{M}\left|y_{i}(k)\right|^{2}
$$

where $M$ means the number of samples in each observation window.

Comparing $v_{i}$ with a predetermined threshold $\lambda$, the state of PU at the $i$ th slot can be determined as follows,

$$
\left\{\begin{array}{ll}
\text { if } v_{i}<\lambda & \text { Accept } H_{0} \\
\text { if } v_{i} \geq \lambda & \text { Accept } H_{1}
\end{array} .\right.
$$

Since $M$ is usually very large, $v_{i}$ approximately obeys Gaussian distribution according to the center limit theorem (CLT) $[27,28]$. Assume that its mean and variance under $H_{0}$ are $m_{0}$ and $\sigma_{0}^{2}$, and that its mean and variance under $H_{1}$ are $m_{1}$ and $\sigma_{1}^{2}$, respectively. Then the distribution of $v_{i}$ is given by

$$
v_{i} \sim\left\{\begin{array}{l}
N\left(m_{0}, \sigma_{0}^{2}\right) H_{0} \\
N\left(m_{1}, \sigma_{1}^{2}\right) H_{1}
\end{array} .\right.
$$

Based on (5), the false alarm probability $P_{f}$ and missed detection probability $P_{m}$ yield to

$$
P_{f}=\int_{\lambda}^{\infty} f\left(v_{i} \mid H_{0}\right) \mathrm{d} v_{i}=Q\left(\frac{\lambda-m_{0}}{\sigma_{0}}\right)
$$




$$
P_{m}=\int_{-\infty}^{\lambda} f\left(v_{i} \mid H_{1}\right) \mathrm{d} v_{i}=1-Q\left(\frac{\lambda-m_{1}}{\sigma_{1}}\right)
$$

where $f\left(v_{i} \mid H_{0}\right)$ and $f\left(v_{i} \mid H_{1}\right)$ are the probability density functions of $v_{i}$ under hypotheses $H_{0}$ and $H_{1}$, respectively; $Q(x)=\int_{x}^{\infty} 1 / \sqrt{2 \pi} e^{-t^{2} / 2} d t$ is the $Q$ function.

\section{MBED Algorithm}

In SS, both false alarm probability and missed detection probability are important. Low false alarm probability is required to guarantee the throughput of $\mathrm{CR}$, and low missed detection probability is needed to ensure the tolerable interference to PU [29]. Unfortunately, these two goals usually contradict with each other. This section firstly re-deduces the threshold of BED algorithm, and then discusses a new algorithm that exploits the Markov model in Fig. 1 to reduce the total cost of false alarm and missed detection.

\subsection{Threshold of BED Algorithm}

BED algorithm jointly considers false alarm and missed detection probabilities, and uses the Bayesian cost as its performance metric. The definition of Bayesian cost can be expressed as [30]

$$
J \triangleq I_{f} P_{0} P_{f}+I_{m} P_{1} P_{m},
$$

where $I_{f}$ and $I_{m}$ are the impact factors of false alarm and missed detection, respectively. Both $I_{f}$ and $I_{m}$ should be positive. [21]

Taking the first-order partial derivative of (8), the threshold of BED algorithm is given by

$$
\begin{aligned}
& \lambda_{\mathrm{B}}=A+B \sqrt{C+2\left(\sigma_{1}^{2}-\sigma_{0}^{2}\right)\left[\ln \left(\frac{P_{0}}{P_{1}}\right)+\ln \left(\frac{I_{f}}{I_{m}}\right)\right]}, \\
& \text { subject to: } C+2\left(\sigma_{1}^{2}-\sigma_{0}^{2}\right)\left[\ln \left(\frac{P_{0}}{P_{1}}\right)+\ln \left(\frac{I_{f}}{I_{m}}\right)\right] \geqslant 0,
\end{aligned}
$$

where $A=\left(m_{0} \sigma_{1}^{2}-m_{1} \sigma_{0}^{2}\right) /\left(\sigma_{1}^{2}-\sigma_{0}^{2}\right), \quad B=\sigma_{0} \sigma_{1} /\left(\sigma_{1}^{2}-\sigma_{0}^{2}\right), \quad C=\left(m_{1}-m_{0}\right)^{2}+2\left(\sigma_{1}^{2}-\sigma_{0}^{2}\right) \ln \left(\sigma_{1} / \sigma_{0}\right)$.

\subsection{Actual Probabilities}

This paper concentrates on the Markovian behavior of PU, as depicted in Fig. 1. According to this characteristic, if the state of PU at the previous slot is explicitly known, the probabilities of PU's state at the current slot can be inferred. More specifically, when the actual state at the previous slot is absence, the actual probabilities of PU's state at the current slot yield to

$$
\left\{\begin{array}{l}
P\left(H_{0}\right)=1-q \\
P\left(H_{1}\right)=q
\end{array}\right.
$$


When the actual state at the previous slot is presence, the actual probabilities of PU's state at the current slot are

$$
\left\{\begin{array}{l}
P\left(H_{0}\right)=p \\
P\left(H_{1}\right)=1-p
\end{array} .\right.
$$

Intuitively, the actual probabilities in (11) and (12) are more precise than the prior probabilities in (1). Therefore, we may use the former to replace the latter in (9). Then the threshold can be rewritten as

$$
\begin{aligned}
& \lambda_{0}=A+B \sqrt{C+2\left(\sigma_{1}^{2}-\sigma_{0}^{2}\right)\left[\ln \left(\frac{1-q}{q}\right)+\ln \left(\frac{I_{f}}{I_{m}}\right)\right]}, \\
& \lambda_{1}=A+B \sqrt{C+2\left(\sigma_{1}^{2}-\sigma_{0}^{2}\right)\left[\ln \left(\frac{p}{1-p}\right)+\ln \left(\frac{I_{f}}{I_{m}}\right)\right]},
\end{aligned}
$$

where $\lambda_{0}$ and $\lambda_{1}$ denote the threshold of current slot when PU's state at the previous slot is $H_{0}$ and $H_{1}$, respectively.

Based on these two thresholds, the performance of SS can be rewritten as

$$
\begin{gathered}
P_{f}=(1-q) Q\left(\frac{\lambda_{0}-m_{0}}{\sigma_{0}}\right)+q Q\left(\frac{\lambda_{1}-m_{0}}{\sigma_{0}}\right) \\
P_{m}=p\left[1-Q\left(\frac{\lambda_{0}-m_{1}}{\sigma_{1}}\right)\right]+(1-p)\left[1-Q\left(\frac{\lambda_{1}-m_{1}}{\sigma_{1}}\right)\right] \\
J=P_{0} I_{f}\left[(1-q) Q\left(\frac{\lambda_{0}-m_{0}}{\sigma_{0}}\right)+q Q\left(\frac{\lambda_{1}-m_{0}}{\sigma_{0}}\right)\right]+P_{1} I_{m} \cdot\left\{p\left[1-Q\left(\frac{\lambda_{0}-m_{1}}{\sigma_{1}}\right)\right]+(1-p)\left[1-Q\left(\frac{\lambda_{1}-m_{1}}{\sigma_{1}}\right)\right]\right\}
\end{gathered}
$$

Without loss of generality, we consider the scenario with equal prior probabilities (namely $p=q)$, and then obtain following proposition.

Proposition 1: The Bayesian cost in (17) is symmetric with respect to the transition probability of $1 / 2$, and reaches the extremum when the transition probability is $1 / 2$.

Proof: The proof of Proposition 1 is given in Appendix A.

Note that when the transition probability is $1 / 2(q=1 / 2)$, we have $\lambda_{0}=\lambda_{1}=\lambda_{B}$, and thus the Bayesian cost in (17) is equal to that in (8). When $q \neq 1 / 2$, the Bayesian cost in (17) is certainly superior. Moreover, if the transition probability is further away from $1 / 2$, the superiority of (17) is more significant. 


\subsection{Description of MBED algorithm}

As illustrated above, if the state of PU at the previous slot is explicitly known, we can choose $\lambda_{0}$ or $\lambda_{1}$ as the threshold accordingly. However, the actual state of PU cannot be obtained in real scenarios. MBED algorithm is proposed to conquer this problem.

Since the detection at the previous slot has been completed, we have a detection result. This result is a measurement of the actual state although it might be erroneous. In MBED algorithm, we use the detection result instead of the actual state. If the detection result is $H_{0}$, we believe that the actual state of the previous slot is $H_{0}$, and choose $\lambda_{0}$ as the threshold at the current slot; otherwise, we choose $\lambda_{1}$.

The detailed steps of MBED algorithm are listed in Algorithm 1.

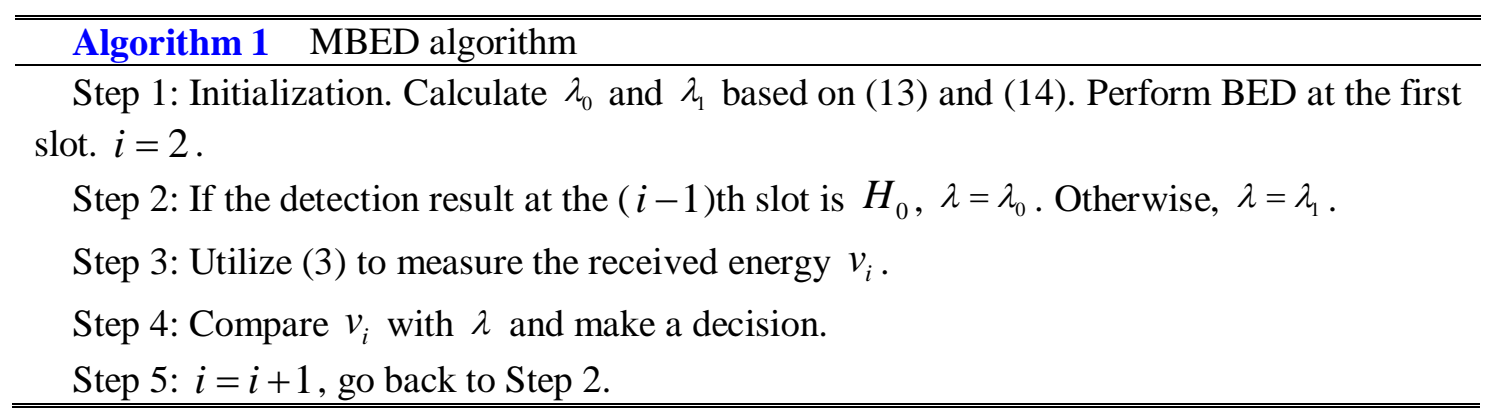

Note that although MBED algorithm updates its threshold at each slot, the new threshold does not need to be calculated. According to Step 2, this algorithm chooses either $\lambda_{0}$ or $\lambda_{1}$, which is obtained at the initialization step. Therefore, it has lower computational complexity compared with other adaptive methods and is more suitable for the real-time SS scenarios in which less calculation time is required.

Furthermore, since this algorithm choose threshold according to detection results, it can improve the performance of SS if detection results are reliable, e.g. in high signal-to-noise ratio (SNR) regions. When SNR is high, its false alarm probability, missed detection probability and Bayesian cost can be approximated by (15), (16) and (17), respectively.

\section{IMBED Algorithm}

MBED algorithm works well if SNR is high. However, when SNR is low, it suffers from great performance degradation because the detection results are probably erroneous. This section proposes an IMBED algorithm to improve the sensing performance in low SNR regions by avoiding adjusting the threshold according to detection results directly.

When implementing SS at the previous slot, we can not only obtain a detection result but also calculate the posterior probability of $H_{x}(x=0,1)$,

$$
P_{x}^{\text {post }}(i-1)=P\left(H_{x} \mid v_{i-1}\right)=\frac{P_{x} \cdot f\left(v_{i-1} \mid H_{x}\right)}{P_{0} \cdot f\left(v_{i-1} \mid H_{0}\right)+P_{1} \cdot f\left(v_{i-1} \mid H_{1}\right)} .
$$

Based on the Markov model in Fig. 1, the probability that PU is absent or present at the current slot can be predicted as 


$$
\left\{\begin{array}{l}
P_{0}^{\text {pred }}(i)=(1-q) P_{0}^{\text {post }}(i-1)+p P_{1}^{\text {post }}(i-1) \\
P_{1}^{\text {pred }}(i)=q P_{0}^{\text {post }}(i-1)+(1-p) P_{1}^{\text {post }}(i-1)
\end{array}\right.
$$

If prediction probabilities $P_{0}^{\text {pred }}(i)$ and $P_{1}^{\text {pred }}(i)$ are more accurate than prior probabilities $P_{0}$ and $P_{1}$, we may utilize them in (9) to obtain an even better threshold [21].

Assuming the equal prior probabilities again, we have following proposition.

Proposition 2: Providing the false alarm and missed detection probabilities at the previous slot are $P_{f}$ and $P_{m}$, respectively. (i) if $q=1 / 2$, prediction probabilities are equal to prior probabilities; (ii) otherwise, prediction probabilities are more accurate than prior probabilities when $P_{f}<1 / 2$ and $P_{m}<1 / 2$.

Proof: The proof of Proposition 2 is given in Appendix B.

Since both $P_{f}<1 / 2$ and $P_{m}<1 / 2$ can be easily satisfied in practical scenarios, prediction probabilities are not worse than prior probabilities. Using the former to replace the latter in (9), a better instantaneous threshold at the $i$ th slot is obtained as

$$
\lambda_{I}(i)=A+B \sqrt{C+2\left(\sigma_{1}^{2}-\sigma_{0}^{2}\right)\left[\ln \left(\frac{P_{0}^{\text {pred }}(i)}{P_{1}^{\text {pred }}(i)}\right)+\ln \left(\frac{I_{f}}{I_{m}}\right)\right]} .
$$

IMBED algorithm is proposed based on the instantaneous threshold above. It first calculates the posterior probabilities at the previous slot, and then predicts the probabilities of PU's states at the current slot. With the prediction probabilities, it re-calculates the threshold of current slot and makes a decision. The detailed steps of IMBED algorithm can be found in Algorithm 2.

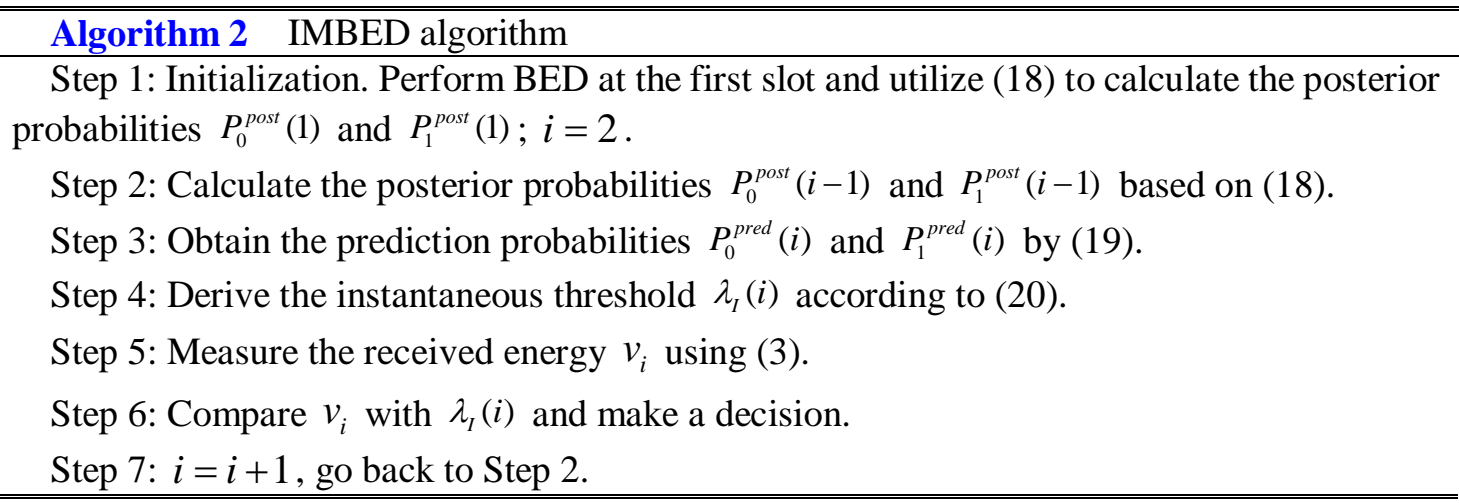

Based on Proposition 2, the sensing performance of IMBED algorithm can be described as follows: (i) when $q=1 / 2$, both IMBED and BED algorithms have the same performance; (ii) when $q \neq 1 / 2$, the performance of IMBED algorithm is certainly better when $P_{f}<1 / 2$ and $P_{m}<1 / 2$. 
Computational complexity of different algorithms is listed in Table 1.

Table 1. Computational complexity of different algorithms

\begin{tabular}{|c|c|c|c|}
\hline Algorithm & Threshold Expression & $\begin{array}{l}\text { When to } \\
\text { Calculate }\end{array}$ & $\begin{array}{c}\text { Computational } \\
\text { Complexity }\end{array}$ \\
\hline BED & $\lambda_{B}=A+B \sqrt{C+2\left(\sigma_{1}^{2}-\sigma_{0}^{2}\right)\left[\ln \left(\frac{P_{0}}{P_{1}}\right)+\ln \left(\frac{I_{f}}{I_{m}}\right)\right]}$ & $\begin{array}{l}\text { once in } \\
\text { Initialization }\end{array}$ & very low \\
\hline MBED & $\begin{array}{l}\lambda_{0}=A+B \sqrt{C+2\left(\sigma_{1}^{2}-\sigma_{0}^{2}\right)\left[\ln \left(\frac{1-q}{q}\right)+\ln \left(\frac{I_{f}}{I_{m}}\right)\right]} \\
\lambda_{1}=A+B \sqrt{C+2\left(\sigma_{1}^{2}-\sigma_{0}^{2}\right)\left[\ln \left(\frac{p}{1-p}\right)+\ln \left(\frac{I_{f}}{I_{m}}\right)\right]}\end{array}$ & $\begin{array}{l}\text { once in } \\
\text { Initialization }\end{array}$ & low \\
\hline IMBED & $\lambda_{I}(i)=A+B \sqrt{C+2\left(\sigma_{1}^{2}-\sigma_{0}^{2}\right)\left[\ln \left(\frac{P_{0}^{\text {pred }}(i)}{P_{1}^{\text {pred }}(i)}\right)+\ln \left(\frac{I_{f}}{I_{m}}\right)\right.}$ & $\begin{array}{l}\text { every time } \\
\text { in each detection }\end{array}$ & high \\
\hline
\end{tabular}

\section{Simulation Results}

In this section, we assume the noise has unit power $\left(\sigma_{n}^{2}=1\right)$. Therefore, $m_{0}=M$, $m_{1}=M(1+\gamma), \sigma_{0}^{2}=2 M, \sigma_{1}^{2}=2 M(1+2 \gamma)$, where $\gamma=\sigma_{s}^{2}$ is the SNR. Moreover, we consider equal priori probabilities $\left(P_{0}=P_{1}\right.$ ), and thus $p=q$. In our simulations, Monte Carlo tests are executed 100000 times, $M=600$, and $I_{f}+I_{m}=2$. ED algorithm derives its threshold based on the constant false alarm probability of 0.1, named constant false-alarm ED (CFED).

Fig. 2 plots the Bayesian cost versus transition probability for $I_{f}=1$ and $S N R=-10 \mathrm{~dB}$. As shown in this figure, transition probability does not affect CFED and BED algorithms because they do not make use of the Markov model. Compared with them, MBED algorithm has lower Bayesian cost, and IMBED algorithm has the lowest Bayesian cost. Note that their Bayesian cost curves are convex and symmetric centered at the transition probability of $1 / 2$, which agrees well with Proposition 1. Moreover, the further transition probability is away from 1/2, the less Bayesian cost is. That is, both MBED and IMBED algorithms are especially superior when PU has a clear tendency of either remaining or changing its state. This phenomenon can be explained as follows. Our algorithms improve sensing performance by inferring the probabilities of PU's states and adjusting the threshold accordingly. If PU tends to be present, we decrease the threshold. On the contrary, if PU tends to be absent, we increase the threshold. However, when the transition probability approaches $1 / 2$, PU will be present or absent equally, and our algorithms make little adjustment on the threshold. 


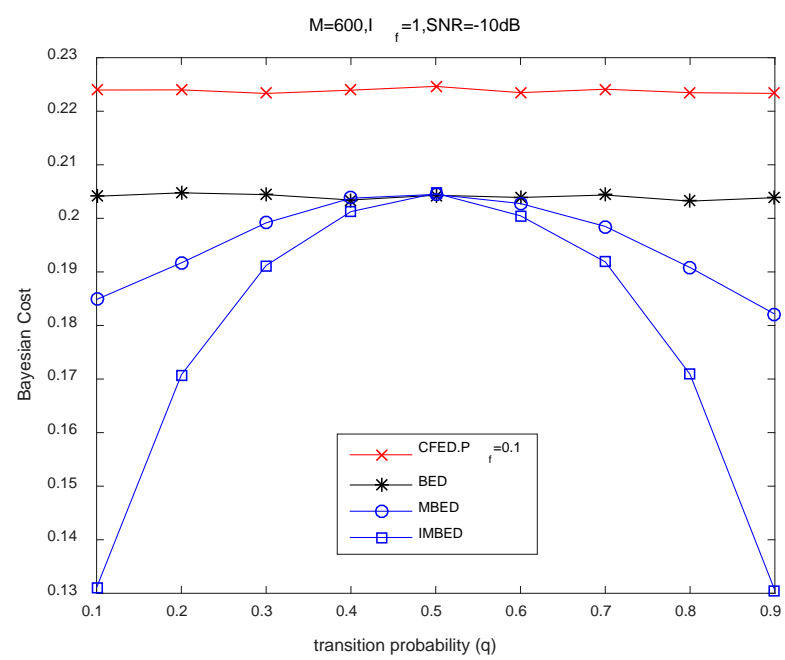

Fig. 2. Bayesian cost against transition probability

In order to investigate the Bayesian cost versus SNR, different transition probabilities are considered: (1) for $q<1 / 2$, we choose $q=0.1$ and 0.2 ; (2) for $q>1 / 2$, we choose $q=0.8$ and 0.9 ; (3) $q=1 / 2$.

Fig. 3 and Fig. 4 demonstrate the curves of Bayesian cost versus SNR for $I_{f}=1$ with transition probabilities $q=0.1,0.9$ and $q=0.2,0.8$, respectively. According to these figures, the Bayesian cost curves of MBED and IMBED algorithms are almost the same when $q=0.1$ and 0.9 , also when $q=0.2$ and 0.8 , which is consistent with Proposition 1 . Note that when SNR is high, MBED algorithm is better than conventional algorithms. However, its Bayesian cost is worse than that of BED algorithm if SNR is below $-10 \mathrm{~dB}$, and even worse than that of CFED algorithm if SNR is below $-11 \mathrm{~dB}$. This may be explained by the facts that the threshold of MBED algorithm depends on detection results, and there are too many errors in detection results when SNR is very low. It can also be seen from these figures that, IMBED algorithm is always superior to all other algorithms, even in low SNR regions. This phenomenon indicates that IMBED algorithm is robust to low SNR and detection errors. For example, in Fig. 3, BED algorithm achieves Bayesian cost of 0.3 at about $\mathrm{SNR}=-12 \mathrm{~dB}$, while IMBED algorithm obtains the same performance at $\mathrm{SNR}=-14 \mathrm{~dB}$. As much as $2 \mathrm{~dB}$ gain can be achieved by use of IMBED algorithm. 

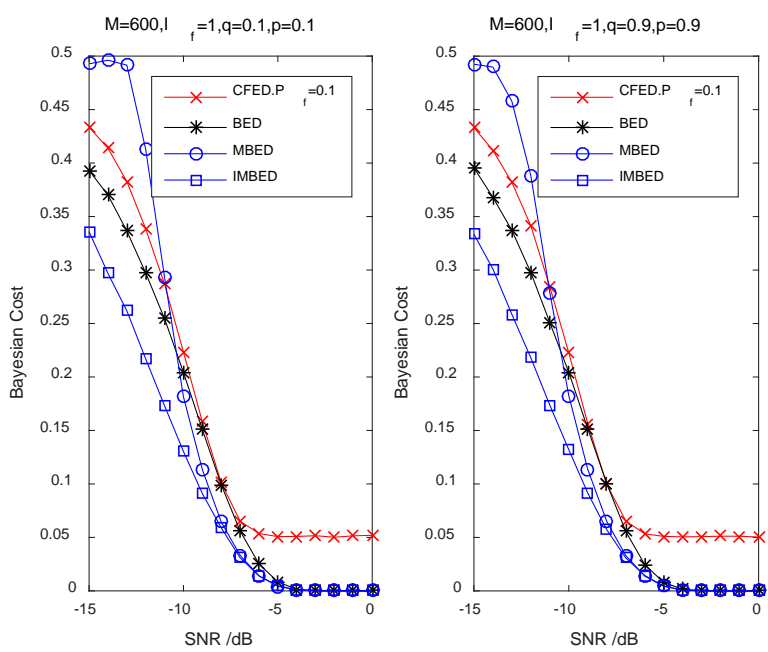

Fig. 3. Bayesian cost against SNR with $q=0.1$ and 0.9
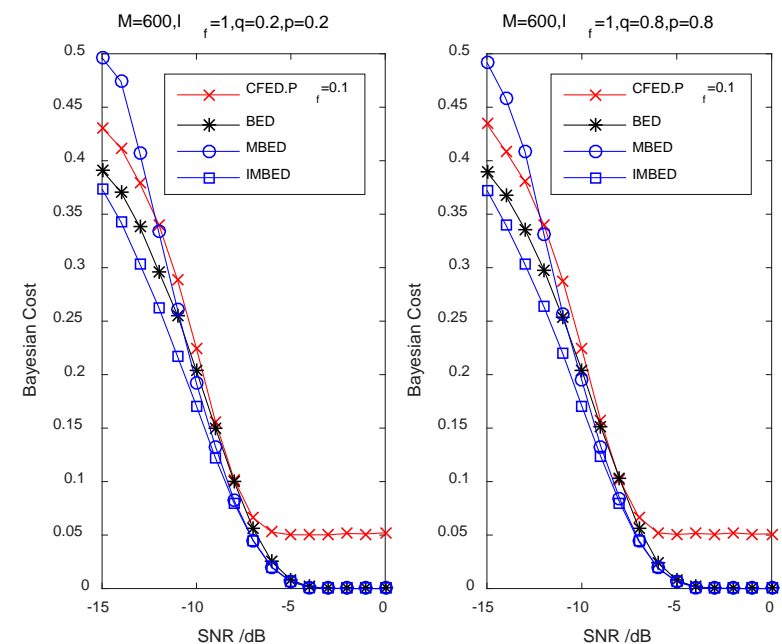

Fig. 4. Bayesian cost against SNR with $q=0.2$ and 0.8

In Fig. 5, the case of $q=1 / 2$ is investigated. As shown in this figure, Bayesian cost of CFED algorithm is highest, and all other curves overlap each other, which means BED, MBED and IMBED algorithms have the same performance. The reason is that, when $q=1 / 2$, there is no potential information about the behavior of PU according to the Markov model, and the thresholds of these algorithms are identical.

Finally, different impacts of false alarm and missed detection are studied. The scenario with $\mathrm{SNR}=-10 \mathrm{~dB}$ and transition probability $q=0.1$ is taken for example in Fig. 6. Because of not considering the impacts of false alarm and missed detection, Bayesian cost of CFED algorithm keeps increasing dramatically as $I_{m} / I_{f}$ increases. BED algorithm is better than CFED algorithm as its threshold is related to $I_{m} / I_{f}$. Compared with BED algorithm, Bayesian cost of MBED algorithm is lower in most regions, but is higher when $I_{m} / I_{f}$ is very large. It is because SU should be extremely conservative and excessively protect PU from interference 
under this circumstance, resulting in too many false alarm errors [31]. Again, Bayesian cost of IMBED algorithm is the lowest, indicating the superiority of this algorithm.

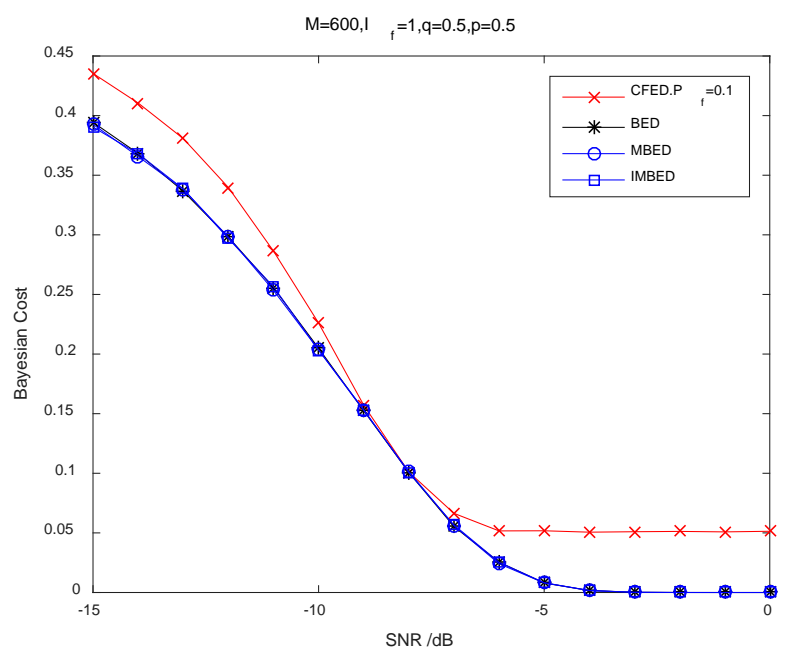

Fig. 5. Bayesian cost against SNR with $q=1 / 2$

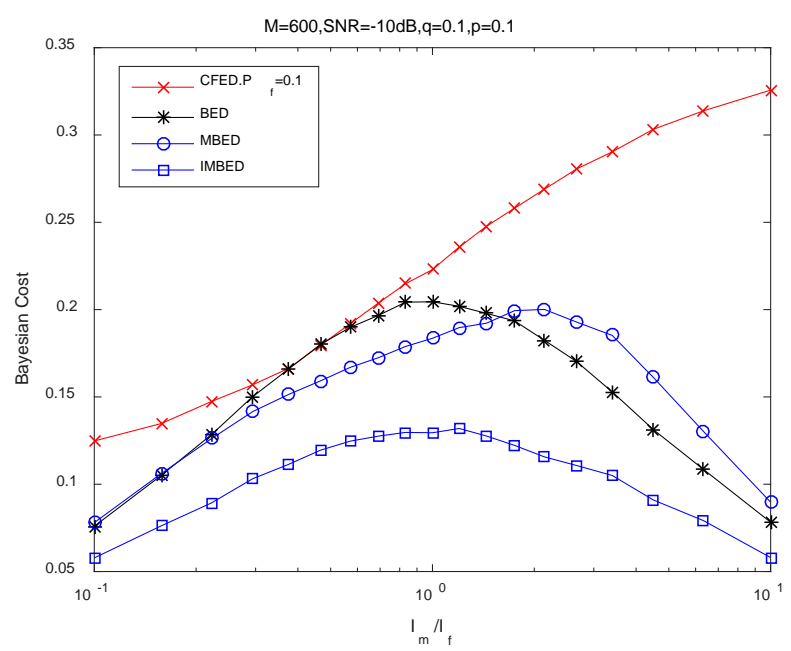

Fig. 6. Bayesian cost against impact of false alarm and missed detection

\section{Conclusion}

This paper has exploited the Markovian behavior of PU to reduce the Bayesian cost of false alarm and missed detection in SS. Two novel adaptive threshold algorithms, including MBED and IMBED, are proposed. MBED algorithm chooses either $\lambda_{0}$ or $\lambda_{1}$ as its threshold at each slot according to the detection result of previous slot. It has low computational complexity and is superior to BED algorithm in high SNR regions. IMBED algorithm re-calculates an instantaneous threshold at each slot based on the posterior probabilities of previous slot. It is able to reduce Bayesian cost more significantly and in a wider SNR region. The conditions under which IMBED algorithm is certainly better than BED algorithm are deduced. Both 
theories and simulation results show that larger performance improvement can be achieved if transition probabilities are further away from 1/2. Based on these two algorithms, higher detection accuracy of TV white spaces can be achieved by exploiting the Markovian behavior of TV broadcasters, which is of key importance to improve the efficiency of TV bands. In the future, the estimation errors of some parameters, such as SNR and transition probabilities, can be investigated.

\section{Appendix A: Proof of Proposition 1}

Feeding $P_{0}=P_{1}$ into (1), $p=q$ is obtained. Consequently, (13), (14) and (17) can be rewritten as

$$
\begin{gathered}
\lambda_{0}(q)=A+B \sqrt{C+2\left(\sigma_{1}^{2}-\sigma_{0}^{2}\right)\left[\ln \left(\frac{1-q}{q}\right)+\ln \left(\frac{I_{f}}{I_{m}}\right)\right]}, \\
\lambda_{1}(q)=A+B \sqrt{C+2\left(\sigma_{1}^{2}-\sigma_{0}^{2}\right)\left[\ln \left(\frac{q}{1-q}\right)+\ln \left(\frac{I_{f}}{I_{m}}\right)\right]}, \\
J(q)=1 / 2 I_{f}(1-q) Q\left(\frac{\lambda_{0}(q)-m_{0}}{\sigma_{0}}\right)+1 / 2 I_{f} q Q\left(\frac{\lambda_{1}(q)-m_{0}}{\sigma_{0}}\right) \\
+1 / 2 I_{m} q\left[1-Q\left(\frac{\lambda_{0}(q)-m_{1}}{\sigma_{1}}\right)\right]+1 / 2 I_{m}(1-q)\left[1-Q\left(\frac{\lambda_{1}(q)-m_{1}}{\sigma_{1}}\right)\right] .
\end{gathered}
$$

Obviously, $\lambda_{0}(q)=\lambda_{1}(1-q), \lambda_{1}(q)=\lambda_{0}(1-q)$. Hence,

$$
\begin{aligned}
J(1-q) & =1 / 2 I_{f} q Q\left(\frac{\lambda_{0}(1-q)-m_{0}}{\sigma_{0}}\right)+1 / 2 I_{f}(1-q) Q\left(\frac{\lambda_{1}(1-q)-m_{0}}{\sigma_{0}}\right) \\
& +1 / 2 I_{m}(1-q)\left[1-Q\left(\frac{\lambda_{0}(1-q)-m_{1}}{\sigma_{1}}\right)\right]+1 / 2 I_{m} q\left[1-Q\left(\frac{\lambda_{1}(1-q)-m_{1}}{\sigma_{1}}\right)\right]=J(q) .
\end{aligned}
$$

Thus, this Bayesian cost is symmetric with the transition probability of $1 / 2$.

Taking the first-order partial derivative of $J(q)$ with respect to $q$, we get

$$
\begin{aligned}
\frac{\partial J}{\partial q} & =\frac{1}{2} I_{f}\left[-Q\left(\frac{\lambda_{0}-m_{0}}{\sigma_{0}}\right)+\frac{2 D \sigma_{1}}{\sqrt{2 \pi} q} e^{-\frac{\left(\lambda_{0}-m_{0}\right)^{2}}{2 \sigma_{0}^{2}}}\right]+\frac{1}{2} I_{f}\left[Q\left(\frac{\lambda_{1}-m_{0}}{\sigma_{0}}\right)-\frac{2 E \sigma_{1}}{\sqrt{2 \pi}(1-q)} e^{-\frac{\left(\lambda_{1}-m_{0}\right)^{2}}{2 \sigma_{0}^{2}}}\right] \\
& +\frac{1}{2} I_{m}\left[-Q\left(\frac{\lambda_{0}-m_{1}}{\sigma_{1}}\right)-\frac{2 D \sigma_{0}}{\sqrt{2 \pi}(1-q)} e^{-\frac{\left(\lambda_{0}-m_{1}\right)^{2}}{2 \sigma_{1}^{2}}}\right]+\frac{1}{2} I_{m}\left[Q\left(\frac{\lambda_{1}-m_{1}}{\sigma_{1}}\right)+\frac{2 E \sigma_{0}}{\sqrt{2 \pi} q} e^{-\frac{\left(\lambda_{1}-m_{1}\right)^{2}}{2 \sigma_{1}^{2}}}\right],
\end{aligned}
$$

where $D=B /\left(\lambda_{0}-A\right), E=B /\left(\lambda_{1}-A\right)$. 
It's obvious that $\partial J /\left.\partial q\right|_{(q=1 / 2)}=0$. Combining with the symmetry, it reaches the extremum when $q=1 / 2$.

\section{Appendix B: Proof of Proposition 2}

In order to compare the accuracy of prior probabilities with that of prediction probabilities, $\alpha_{x}$ is defined as the gap between prior probabilities and actual probabilities at the current slot when the previous state is $H_{x}$, and $\beta_{x}$ is defined as the gap between prediction probabilities and actual probabilities at the current slot when the previous state is $H_{x}$, where $x=0,1$. Obviously, the smaller gap is, the more accurate probabilities are.

When the previous state is $H_{0}$ (PU is absent at the previous slot), the actual probability of $H_{1}$ at the current slot is $q$ according to Fig. 1. Moreover, since the false alarm probability at the previous slot is $P_{f}$, prediction probabilities can be written as

$$
\left\{\begin{array}{l}
P_{0}^{\text {pred }}(i)=(1-q)\left(1-P_{f}\right)+p P_{f} \\
P_{1}^{\text {pred }}(i)=q\left(1-P_{f}\right)+(1-p) P_{f}
\end{array} .\right.
$$

Considering $P_{0}=P_{1}$ and thus $p=q$, the gaps can be derived as follows,

$$
\begin{gathered}
\alpha_{0}=\left|\frac{1}{2}-q\right|=\left|\frac{1}{2}(1-2 q)\right|, \\
\beta_{0}=\left|q\left(1-P_{f}\right)+(1-p) P_{f}-q\right|=\left|P_{f}(1-2 q)\right| .
\end{gathered}
$$

So, if $q=1 / 2$, we have $\beta_{0}=\alpha_{0}$; otherwise, we have $\beta_{0}<\alpha_{0}$ on condition of $P_{f}<1 / 2$.

Similarly, when the previous state is $H_{1}$ (PU is present at the previous slot), prediction probabilities can be derived as

$$
\left\{\begin{array}{l}
P_{0}^{\text {pred }}(i)=(1-q) P_{m}+p\left(1-P_{m}\right) \\
P_{1}^{\text {pred }}(i)=q P_{m}+(1-p)\left(1-P_{m}\right)
\end{array},\right.
$$

and the gaps are given by

$$
\begin{gathered}
\alpha_{1}=\left|\frac{1}{2}-p\right|=\left|\frac{1}{2}(1-2 q)\right|, \\
\beta_{1}=\left|(1-q) P_{m}+p\left(1-P_{m}\right)-p\right|=\left|P_{m}(1-2 q)\right| .
\end{gathered}
$$

Obviously, $\beta_{1}$ equals to $\alpha_{1}$ on condition of $q=1 / 2$, and $\beta_{1}$ is smaller than $\alpha_{1}$ on condition of $P_{m}<1 / 2, q \neq 1 / 2$.

Consequently, if $q=1 / 2$, prediction probabilities are certainly equal to prior probabilities; otherwise, prediction probabilities are more accurate than prior probabilities when $P_{f}<1 / 2$ and $P_{m}<1 / 2$. 


\section{Acknowledgment}

The authors would like to thank the Nature Science Foundation of China (Grant No. 61201264, 61362018, 61401165), China Scholarship Council (Grant No. 201508350023), Jiangsu Province Postdoctoral Scientific Research Project (Grant No. 1402041B), Scientific Research Fund of Hunan Provincial Education Department (Grant No. 16A174), and Huaqiao University (Grant No. 13BS101) for their financial supports.

\section{References}

[1] S. Filin, T. Baykas, H. Harada, F. Kojima, and H. Yano, "IEEE Standard 802.19.1 for TV white space coexistence," IEEE Communications Magazine, vol. 54, no. 3, pp. 22-26, 2016. Article (CrossRef Link)

[2] C.-S. Sum et al., "Cognitive communication in TV white spaces: An Overview of Regulations, Standards, and Technology," IEEE Commun. Mag., vol. 51, no. 7, pp. 138-145, 2013. Article (CrossRef Link)

[3] J. Mitola and G. Q. Maguire, “Cognitive radio: making software radios more personal,” IEEE personal communications, vol. 6, no. 4, pp. 13-18, 1999. Article (CrossRef Link)

[4] S. Haykin, "Cognitive radio: brain-empowered wireless communications," IEEE journal on selected areas in communications, vol. 23, no. 2, pp. 201-220, 2005. Article (CrossRef Link)

[5] S. Haykin, D. J. Thomson, and J. H. Reed, "Spectrum sensing for cognitive radio," Proceedings of the IEEE, vol. 97, no. 5, pp. 849-877, 2009. Article (CrossRef Link)

[6] P. D. Sutton, K. E. Nolan, and L. E. Doyle, "Cyclostationary signatures in practical cognitive radio applications,” IEEE Journal on selected areas in Communications, vol. 26, no. 1, pp. 13-24, 2008. Article (CrossRef Link)

[7] V. I. Kostylev, "Energy detection of a signal with random amplitude," in Proc. of IEEE International Conference on Communications (ICC 2002), pp. 1606-1610, 2002. Article (CrossRef Link)

[8] H. Urkowitz, "Energy detection of unknown deterministic signals," Proceedings of the IEEE, vol. 55, no. 4, pp. 523-531, 1967. Article (CrossRef Link)

[9] R. Tandra and A. Sahai, "SNR Walls for Signal Detection," IEEE J. Sel. Topics Signal Process., vol. 2, no. 1, pp. 4-17, 2008. Article (CrossRef Link)

[10] Y. Zou, Y.-D. Yao, and B. Zheng, "Cooperative relay techniques for cognitive radio systems: Spectrum sensing and secondary user transmissions," IEEE Commun. Mag., vol. 50, no. 4, pp. 98-103, 2012. Article (CrossRef Link)

[11] W. Choi, M.-G. Song, J. Ahn, and G.-H. Im, "Soft Combining for Cooperative Spectrum Sensing over Fast-Fading Channels," IEEE Commun. Lett., vol. 18, no. 2, pp. 193-196, 2014. Article (CrossRef Link)

[12] W. Han, J. Li, Z. Li, J. Si, and Y. Zhang, "Efficient Soft Decision Fusion Rule in Cooperative Spectrum Sensing," IEEE Trans. Signal Process., vol. 61, no. 8, pp. 1931-1943, 2013. Article (CrossRef Link)

[13] G. Verma and O. Sahu, "Intelligent selection of threshold in cognitive radio system," Telecommunication Systems, pp. 1-10, 2016. Article (CrossRef Link)

[14] M. López-Benítez and F. Casadevall, "Improved energy detection spectrum sensing for cognitive radio,” IET communications, vol. 6, no. 8, pp. 785-796, 2012. Article (CrossRef Link)

[15] M. Kist, L. R. Faganello, L. Bondan, M. A. Marotta, L. Z. Granville, J. Rochol, and C. B. Both, "Adaptive threshold architecture for spectrum sensing in public safety radio channels," in Proc. of 2015 IEEE Wireless Communications and Networking Conference (WCNC 2015), pp. 287-292, 2015. Article (CrossRef Link) 
[16] H.-H. Choi, K. Jang, and Y. Cheong, "Adaptive sensing threshold control based on transmission power in cognitive radio systems," in Proc. of 2008 3rd International Conference on Cognitive Radio Oriented Wireless Networks and Communications (CrownCom 2008), pp.1-6, 2008.

Article (CrossRef Link)

[17] Z. Bao, B. Wu, P.-H. Ho, and X. Ling, "Adaptive threshold control for energy detection based spectrum sensing in cognitive radio networks," in Proc. of IEEE Global Telecommunications Conference (GLOBECOM 2011), pp. 1-5, 2011. Article (CrossRef Link)

[18] X. Ling, B. Wu, H. Wen, P.-H. Ho, Z. Bao, and L. Pan, "Adaptive threshold control for energy detection based spectrum sensing in cognitive radios," IEEE Wireless Communications Letters, vol. 1, no. 5, pp. 448-451, 2012. Article (CrossRef Link)

[19] S. Zhang and Z. Bao, “An adaptive spectrum sensing algorithm under noise uncertainty,” in Proc. of 2011 IEEE International Conference on Communications (ICC 2011), pp. 1-5, 2011. Article (CrossRef Link)

[20] N. Wang, Y. Gao, and X. Zhang, “Adaptive spectrum sensing algorithm under different primary user utilizations,” IEEE Communications Letters, vol. 17, no. 9, pp. 1838-1841, 2013. Article (CrossRef Link)

[21] S. Peng, X. Yang, S. Shu, and X. Cao, "Exploitation of temporal persistence for accuracy improvement in primary user detection,” IET communications, vol. 4, no. 15, pp. 1855-1864, 2010. Article (CrossRef Link)

[22] Q. Zhao, L. Tong, and A. Swami, “Decentralized cognitive mac for dynamic spectrum access,” in Proc. of the First IEEE International Symposium on New Frontiers in Dynamic Spectrum Access Networks (DySPAN 2005), pp. 224-232, 2005. Article (CrossRef Link)

[23] Q. Zhao, L. Tong, A. Swami, and Y. Chen, "Decentralized cognitive MAC for opportunistic spectrum access in ad hoc networks: A POMDP framework,” IEEE Journal on selected areas in communications, vol. 25, no. 3, pp.589-600, 2007. Article (CrossRef Link)

[24] S. Geirhofer, L. Tong, and B. M. Sadler, "A measurement-based model for dynamic spectrum access in wlan channels," in Proc. of 2006 IEEE Military Communications conference (MILCOM 2006), pp. 1-7, 2006. Article (CrossRef Link)

[25] S. Geirhofer, L. Tong, and B. M. Sadler, "Dynamic spectrum access in WLAN channels: Empirical model and its stochastic analysis," in Proc. of the first international workshop on Technology and policy for accessing spectrum, p. 14, 2006. Article (CrossRef Link)

[26] Q. Zhao and B. Krishnamachari, "Structure and optimality of myopic sensing for opportunistic spectrum access," in Proc. of 2007 IEEE International Conference on Communications (ICC 2007), pp. 6476-6481, 2007. Article (CrossRef Link)

[27] J. Ma, G. Zhao, and Y. Li, "Soft combination and detection for cooperative spectrum sensing in cognitive radio networks," IEEE Transactions on Wireless Communications, vol. 7, no. 11, pp. 4502-4507, 2008. Article (CrossRef Link)

[28] L. Rugini, P. Banelli, and G. Leus, "Small sample size performance of the energy detector," IEEE Communications Letters, vol. 17, no. 9, pp. 1814-1817, 2013. Article (CrossRef Link)

[29] P. R. Nair, A. P. Vinod, and A. K. Krishna, "An adaptive threshold based energy detector for spectrum sensing in cognitive radios at low SNR," in Proc. of IEEE International Conference on Communication Systems, pp. 574 -578, 2010. Article (CrossRef Link)

[30] S.-C. Hong, M. H. Vu, and V. Tarokh, "Cognitive sensing based on side information," in Proc. of 2008 IEEE Sarnoff Symposium, pp. 1-6, 2008. Article (CrossRef Link)

[31] Q. Zhao and B. M. Sadler, "A survey of dynamic spectrum access," IEEE Signal Processing Magazine, vol. 24, no. 3, pp. 79-89, 2007. Article (CrossRef Link) 


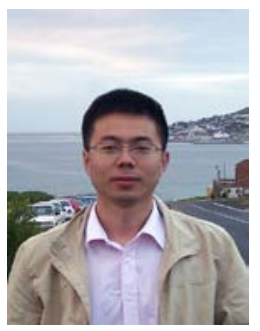

Shengliang Peng received his Ph.D. degree in electric engineering from the Nation Mobile Communications Laboratory, Southeast University, China, in 2011. After that, he joined the School of Information Science and Engineering, Huaqiao University, China, as an assistant professor. From 2016 to 2017, he was a visiting research scholar with the department of electrical and computer engineering, Stevens Institute of Technology, USA. Now he is an associate professor at Huaqiao University. His research interests include cognitive radio, low power wide area communications, and deep learning.

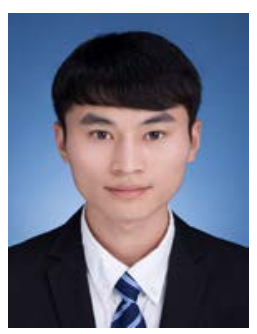

Renyang Gao received his B.S. and M.S. degrees in Communications Engineering from Huaqiao University in 2014 and 2017, respectively. His research interest is in wireless communications.

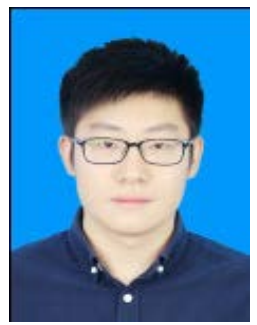

Weibin Zheng received his B.S. degree in Communications Engineering from Huaqiao University in 2015. He is currently pursuing his M.S. degree with the College of Information Science and Engineering, Huaqiao University. His research interests include cognitive radio and deep learning.

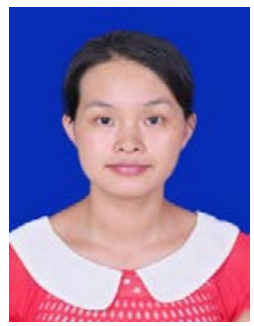

Kejun Lei is a Ph. D. candidate of Hunan University in China. Her research interests include signal detection and estimation algorithms, smart grid and spectrum sensing in cognitive radio. 